\title{
Research on the anti habitual violation of power enterprises
}

\author{
Zhu Ming, Li Fei, Zhou Xuelong \\ Benxi Power Supply Company of National Grid Liaoning Electric Power Co., Ltd. \\ bxwl_650928@126.com
}

Keywords: Anti-violation; Power Enterprise; Countermeasure; Focus

\begin{abstract}
This paper studies and analyzes the current work situation of anti-violation of power enterprises, seeks the basic reasons for the poor effect of anti-violation, clarifies the three effective countermeasures of creating favorable environment, improving employee safety and eliminating management defects, and discusses the specific focuses of the work.
\end{abstract}

\section{Introduction}

Whether for power enterprises or power construction enterprises, safety production has been the priority of all power work. However, with the rapid development of power grid construction nowadays, the violations leading to all kinds of safety accidents occur frequently, and all kinds of problems in electric power construction appear ceaselessly, making constantly reflection and research on violations.

\section{Necessity of anti-violation}

Anti-violation is a systematic project, which cannot do without the participation and support of leadership at different levels, every department, employees of all departments and their families, or the cooperation of all departments, so the leaders' attention is the key.

Electric power construction safety and anti-violation have been the key content of the safety supervision and management of power grid corporations at all levels among power enterprises, and the anti-violation action has been launched in various departments of power enterprises in full swing. And the safety management supervising personnel of power construction enterprises must be well aware of the difficulties and complexity of anti-violation of the power construction enterprises.

Now, whether it is in the production site or in the production offices, a relatively strong safety atmosphere can be perceived, and the anti-violation has been effective and has a good internal and external environment. Anti-violation must adhere to be people-oriented, proceed from the perspective of caring for the staff, and take the training of employees' safety awareness, basic skills and self safety protection skills as the starting point and goal of anti-violation.

The anti-violation work should be done wholeheartedly and persistently, and dare to crack down the violation behaviors. Besides, it should constantly sum up experiences, make improvement, and combine not only toughness and softness, but also blocking and leading.

\section{“Six combinations" supposed to be done by anti-violation}

Maintaining safety is the premise and basis for power enterprises to do a good job in various production works, and an important measure to improve the safety management of power supply enterprises. To avoid the emergence of a variety of violations plays a vital role in the daily production process. "Anti-violation" should do the following "six combinations".

First, it should be combined with high awards and heavy punishment. All levels of safety management personnel should increase the supervision intensity, and the violated staff should be publicly exposed and punished according to the law, while advanced units or individuals with outstanding achievements in safety production should be awarded regularly or irregularly. High awards and heavy punishment are combined and implemented strictly, sincerely making the safety requirements enshrined in the hearts of the staff. 
Second, it should be combined with creating a safety production culture. Through the development of all kinds of publicity and education activities, the companies should enhance staff's sense of responsibility to develop their good habit of observing regulations and disciplines, remind and stop violation acts timely, and jointly create a good culture of safety production.

Third, it should be combined with the training of strengthening employees' basic skills. "Anti-violation" lectures should be delivered, technical training be strengthened, and learning and test are organized, so that the comprehensive quality and technical level of the staff will be continuously improved, and the staff will give full play of the principle role in safety.

Fourth, it should be combined with the safety production responsibility system. The sense of responsibility of all employees should be strengthened, labor and work disciplines be tightened, the standardized operations be fully implemented, and the safety responsibility system in all levels be further committed, making "standard posts and standard assignments" be the conscious action of each employee.

Fifth, it should be combined with the safety inspections in spring and autumn. For the high occurrence links of violations in the field of production maintenance, operation, construction debugging, and multi-operations of rural power, the inherent or potential risk on the whole process or some operation process should be analyzed timely, and effective measures be taken to curb the trend of high occurrence of violation.

Sixth, it should be combined with strengthening the safety management quality of the team. The violation rules of "striking hard" and "punishing severe" in the grass root team should be established, violation behaviors be probed thoroughly, violation reasons and consequences be analyzed and suggestions to terminate the violations be proposed, so as to widely build "anti-violation" mass base.

\section{Strengthen safety management}

(1) The work members should fully fulfill the responsibilities of mutual supervision, and remind and care each other frequently during working to tighten the safety conscience, and put an end to the negative attitude of the carelessness or absent-mind.

(2) The executive force should be strengthened and the safety rules and regulations be fully implemented. Only a sound safety management system can ensure the safe mechanism long-term stable operation. The assessment force is enhanced, so that the "three irons" will be really implemented to oppose "three violations"; the punishment force is enhanced, so that people will take safety rules as law and meticulously perform them. The uncovered habitual violations should be immediately educated and punished.

(3) The effect of the meeting before work should be noticed. Each time before work, the corresponding professional technical training should be done according to the work content, and the corresponding standardized work procedures be learned, enabling each member to have a clear understanding of the requirements of the standard cards and the contents, procedures and standards of every work, and the dangerous points and pre-control measures, so as to ensure the safety and put an end to the violation.

(4) The provisions of "anti six don't", "three combats and ten shoulds", "six eliminates" developed by the state grid corporation should be earnestly carried out, and the basic management of safety work be strengthened practically. The "no violation group" activities are to be developed, where the general staff are mobilized from the bottom up based on safety production standardization and management science. Team performance is assessed, starting from the control of operational violation, management violation, installation violation and command violation, and focusing on opposing the individual violation behaviors.

(5) Safety activity, which is the platform for the centralized exchange of all members, cannot be a mere formality. The problems found in their respective work can be collected and spoken out in safety activities, so as to make clear to everyone of risks in the device, and violations in the work, and discuss how to solve these problems and eliminate potential safety problems and violations. 
(6) Through the massed learning of accident newsflash and safety briefing, the anti-violation awareness is strengthened. During learning, it is necessary to avoid rigidity and pay attention to the data collection and sorting and analysis of typical cases, and have a discussion and analysis combined with the work experiences. It is feasible to take others' accident as own, do in-depth analysis of the cause of the accident, and warn each member combining with the practical work of the team, so as to continuously improve everyone's safety awareness, and strengthen the ability of accident prevention.

(7) The personnel responsibility system should be earnestly implemented, so that each person is responsible for his own work completely.

\section{Suggestions on the further improvement of the anti-violation effect}

(1) The safety assurance system and safety supervision system should be combined organically, and supervision system must carry out overall and all-around supervision and eliminate random, and at the same time, it should urge the safety assurance system to fully achieve pre command, pre thinking, and pre caution, to improve safety management level solidly.

(2) Safety production management work should be fully participated by all staff. Safety production management is not just the responsibility of the leadership and management, but need the full participation of the staff. Safety pressure should be transferred level upon level, and safety index be shared by everyone, so as to achieve the phenomenon where individual is responsible for the safety of the team, team for the safety of power supply bureau, and power supply bureau for the safety of electric power bureau. Employees in the bureau should conscientiously implement the safety regulations, do every assignment well with the attitude of being responsible for enterprises, individuals and families, and create a safety culture of "pride in observing regulations and shame in violation " through joint efforts.

(3)Management should be familiar with the work habits and violation history of the team, and the violation record and recognition of every staff. In this way, they know well of the management and supervision of the team, and are targeted and focused in anti-violation.

(4) The leadership should not be blindly optimistic, but have the crisis awareness on production safety. There is just a step away between violation and safety accidents, which should be clearly noticed. In order to put an end to persistence and repetition of violation, anti- violation must be persevered. The safety and anti-violation are not noticed until there are accidents, which should be firmly opposed.

\section{Anti-violation measures}

In short, "anti-violation" is a long-term and arduous task, which is worth a long-term research and discussion. Power supply enterprises should continue to bring forth work ideas, change the way of work, take strong measures to resolve the current problems and the roots, build the first defense of safety production, and strive to achieve safety production controllable, so as to ensure the long-term stability of safety production.

To achieve safety management, an effective safety supervision mechanism must be established. Each team should carry out from the actual situation of itself when establishing safety supervision posts. A few high quality employees with rich work experiences are selected from all members of the team as the safety supervisors, so that there is at least one safety supervisor in the production site to assume the responsibility of standardizing the operators' behaviors in the team. Also often organizing team members to participate in safety learning exchange is also an effective measure. In safety exchange, others' safety accident serves as a lesson, and one can remind himself by learning from others' lessons.

As for enterprises, the responsibility of preventing accidents is weightier than Mount Tai. More prevention leads to fewer accidents, so taking preventive measures is extremely important. 


\section{Reference}

[1] The eighteen major anti accident measures of China State Grid Corpoaration [Z].

[2] Construction management [Z].

[3] The collection of provisions of the national power grid security management [Z].

[4] Tian Yuping. Questions and answers of anti-violation of power enterprises [M]. Beijing: China Electric Power Press, 2008

[5] Li Donglai, Zhu Jianjun. Rules and prevention of violation [J]. Chinese electric power enterprise management.2010.

[6] Huang Xianhua. Analysis of the application of anti-violation in electric power production safety management [J]. Chinese power education, 2009 